\title{
Sistema Nacional de Rehabilitación Integral: Limitaciones en el concepto, el marco normativo y la formación profesional ${ }^{1}$
}

\author{
National System of Integral Rehabilitation: limitations on the \\ concept, the regulatory framework and training
}

Nancy Jeanet Molina Achury²

Recibido: 14 de junio 2016 • Aceptado: 19 de julio de 2016

Molina, N. (2016). Sistema Nacional de Rehabilitación Integral: limitaciones en el concepto, el marco normativo y la formación profesional. Revista Ocupación Humana, 16 (1), 91-99.

Resumen: Esta reflexión trata sobre tres aspectos. En primera instancia, se afirma la inexistencia de un Sistema Nacional de Rehabilitación Integral; en su lugar, se caracterizan una serie de cuestiones que, a juicio de la autora, ocupan el espacio político de esta ausencia, convirtiéndose en una distracción en relación a un debate central en nuestra sociedad. En segundo lugar, se identifican aspectos que están en la base de esta situación y que se sintetizan en los mecanismos de permanencia de lógicas hegemónicas que terminan excluyendo a un grupo poblacional de ser objeto de políticas públicas que enfrenten las inequidades estructurales de un sistema en donde, la lógica de la funcionalidad para la productividad, es un aspecto central. En tercer lugar, se profundiza sobre el vínculo formación profesional - reproducción de lógicas de dominación y exclusión, se identifican algunos de los aspectos centrales que perpetúan el estado de cosas que contribuyen a la ausencia misma del sistema, y se sugieren algunos elementos que podrían promover un proceso de cambio en la formación profesional y contribuir a la definición de un Sistema Nacional de Rehabilitación.

Palabras clave: rehabilitación, política pública, formación profesional.

\begin{abstract}
This reflection is about three aspects. First, it states that there is an absence of a National System of Integral Rehabilitation. Instead, there is a characterization of a number of issues which, in the opinion of the author, occupy the political space of such absence, becoming a distraction in relation to a central debate in our society. Second, there is an identification of the aspects that form the basis of this situation and that are synthesized in the mechanisms of continuity of the hegemonic logic that ends up excluding one population group from being recipient of public policies that address the structural inequities of a system in which the logic of functionality for productivity is a central aspect. Third, it delves into the link that exists between professional training and the reproduction of domination and exclusion logics; identifies some of the central aspects that perpetuate the state of affairs that contribute to the very absence of the system, and suggests some elements that might promote a process of change in the professional training so that it contributes to the definition of a National Rehabilitation System.
\end{abstract}

Key words: rehabilitation, public policy, professional education.

\footnotetext{
${ }^{1}$ Este artículo se deriva de la ponencia presentada por la autora el 5 de marzo de 2016 en la ciudad de MedeIlín, en el marco del XVI Congreso Colombiano de Terapia Ocupacional.

${ }^{2}$ Fisioterapeuta. Magíster en Ciencias de la Salud del Trabajo. Doctora en Ciencias. Profesora Asociada, Universidad Nacional de Colombia.njmolinaa@unal.edu.co
} 


\section{La inexistencia del Sistema Nacional de Rehabilitacion Integral}

La página web del Ministerio de Salud refiere que:

"el concepto que se maneja en Colombia respecto a la rehabilitación integral, establece la visión multidimensional y biosicosocial de las personas con discapacidad, lo que implica la provisión continua y coherente de acciones dirigidas al individuo, a su familia y a su comunidad, desarrolladas en corresponsabilidad por los diferentes sectores: salud, educación, trabajo, cultura, recreación y deportes, comunicaciones y transporte, entre otros, con el objeto de facilitar la promoción, prevención, recuperación, rehabilitación e inclusión social ${ }^{3}$ de la población." (MinSalud, 2015).

Sin embargo, la Ley 1618 de 2013 define la rehabilitación integral como "mejoramiento de la calidad de vida y la plena integración de la persona con discapacidad al medio familiar, social y ocupacional, a través de procesos terapéuticos, educativos y formativos que se brindan acorde al tipo de discapacidad" (Gobierno de la República de Colombia, 2013).

Esta definición proyecta de forma clara tres aspectos centrales de la construcción social en torno a la rehabilitación como respuesta a la discapacidad. El primero ratifica la idea establecida de que la discapacidad es un asunto que se define en el plano de los individuos, con énfasis en la dimensión biológica, particularmente centrada en la deficiencia / limitación. Segundo, en coherencia con lo anterior, proyecta el ámbito de la intervención a la familia y a la comunidad, no como expresión de la dinámica social, sino en vínculo instrumental de escenarios relacionales del individuo. Tercero, separa claramente la idea de rehabilitación funcional ${ }^{4}$ de la de inclusión social, siendo la rehabilitación integral la suma de las dos anteriores.

Esta interpretación se ratifica en diversos estudios que analizan los contenidos de las políticas públicas en Colombia. Así, en palabras de Angarita (2014), el análisis de los documentos normativos muestra:

una tendencia significativa hacia el modelo individual, con ambigüedad en la terminología utilizada que tiende a ser peyorativa y reactiva, (más evidente en normas anteriores a 2009) donde contenidos latentes se inclinan hacia la exclusión a partir del reforzamiento de estereotipos con una fuerte propensión a fomentar la adaptación individual al medio y a situaciones vitales de las personas con discapacidad. (...) Al ser el modelo individual el imperante en el marco nor-

\footnotetext{
3 "Es un proceso que asegura que todas las personas tengan las mismas oportunidades, y la posibilidad real y efectiva de acceder, participar, relacionarse y disfrutar de un bien, servicio o ambiente, junto con los demás ciudadanos, sin ninguna limitación o restricción por motivo de discapacidad, mediante acciones concretas que ayuden a mejorar la calidad de vida de las personas con discapacidad" Ley 1618 de 2013. Art. 2, núm. 7. (Gobierno de la República de Colombia, 2013).

4 "Es el proceso de acciones médicas y terapéuticas encaminadas a lograr que las personas con discapacidad estén en condiciones de alcanzar y mantener un estado funcional óptimo desde el punto de vista físico, sensorial, intelectual, psíquico o social, de manera que les posibilite modificar su propia vida y ser más independientes" Ley 1618 de 2013, Art. 2, núm. 6. (Gobierno de la República de Colombia, 2013).
} 
mativo de apoyo a la discapacidad, las respuestas se centraron en el aspecto capacidad de funcionar, siendo el aspecto de oportunidad de elección el menos percibido (p. 87).

El país cuenta entonces con un acumulado de normas, planes y políticas de discapacidad que se vinculan al Sistema Nacional de Discapacidad (Ley 1145 de 2007 - Congreso de la República, 2007) y la Política Pública de Discapacidad e Inclusión Social (CONPES 166 de 2013 - Departamento Nacional de Planeación, 2013), los cuales muestran una cierta evolución en los conceptos, que sin embargo, denotan la ausencia de una visión de rehabilitación integral y de un sistema articulado, con procesos actualizados y continuos (Molina-Achury et al., 2016).

Así mismo, se plantea el poco impacto en el campo de la garantía de los derechos: se mantiene una lógica focalizada y fragmentada de respuestas a las necesidades de la población en materia de servicios de rehabilitación, caracterizadas por acciones de corte sectorial, sin una mirada integral del sujeto y sus necesidades, quien recibe del Estado beneficios por una condición particular, pero no por el conjunto de sus necesidades (Molina-Achury et al., 2016).

Con todo, se hace evidente el marcado individualismo en el concepto de proceso de rehabilitación integral, el cual le debe devolver a un sujeto, que recibe muchos servicios y de todas partes (la suma de todos), el volver a tener lo que se perdió o se debió tener. Así mismo, se identifica un concepto pasivo en relación a los sujetos que son "intervenidos". Esto se relaciona también con esa peligrosa escisión que se ha hecho entre el cuerpo funcional, la deficiencia, y el cuerpo social, la participación. Pareciera, desafortunadamente, que la entrada de la comprensión social de la discapacidad se asumiera como antagónica -excluyente- opuesta a las acciones de rehabilitación, poniendo en contraposición el componente médico-rehabilitador, frente al modelo social-de inclusión. En este mismo sentido, se observa una dicotomía entre intervención del cuerpo e intervención social, como si el cuerpo no fuese un elemento de la socialidad humana.

En términos de las prácticas concretas, particularmente en el sector salud, la manera de operar del sistema de salud favorece la sumatoria de acciones desarrolladas por distintos profesionales, centradas en lo funcional, que privilegian la realización de terapias. En este marco, la oferta de servicios no responde efectivamente a las necesidades de la población. Se reconoce la influencia de factores que son determinantes en la prestación de servicios de rehabilitación, como lo constituyen la focalización de la oferta a algunos grupos, en detrimento de una prestación de servicios equitativa; la falta de estándares de calidad y el limitado uso de procedimientos estandarizados; los escasos o nulos mecanismos de evaluación o seguimiento, y el limitado sistema de verificación del impacto logrado con las acciones.

Estos elementos se resumen en la idea presentada por María Teresa Buitrago (2013) en su tesis doctoral titulada: Discapacidades peregrinas. Construc- 
ciones sociales de la discapacidad en Colombia: aportes para Salud Pública desde una perspectiva crítica, quien analiza el período 1970 - 2010 y plantea:

Los resultados mostraron una irrupción retórica del marco de derechos en la normativa sin respuestas concretas, la hegemonía de las representaciones desde el modelo biomédico, al igual que respuestas ancladas en la rehabilitación funcional. Las instituciones pasaron de la atención de beneficencia -caridad- a un modelo subsumido por la lógica neoliberal de prestación de servicios. Los principales desafíos para la Salud Pública son: ampliar el espectro de sus acciones, trascender la representación biomédica y optar por abordajes interdisciplinarios e intersectoriales que aboguen por la emancipación de esta población, basados en la pluralidad de saberes y maneras dignas de estar en el mundo, más allá de la mirada funcional del desarrollo y bienestar, que lo equipara a la productividad y los beneficios materiales que la misma provee (p. IX).

\section{Elementos constitutivos de esta situación}

En primer lugar, como se ha evidenciado, la rehabilitación se atrapa en el marco de la discapacidad y hereda las limitaciones de las visiones y prácticas construidas, las cuales mayoritariamente se centran en el sujeto y en la discapacidad como limitación, lo que distancia y desvanece el debate de las causas estructurales que la producen, tales como el no reconocimiento de la diversidad de capacidades o la naturalización de arreglos sociales que ge- neran desigualdades e inequidades de forma sistemática, como mecanismo para garantizar la acumulación económica y el poder de unos grupos sobre otros: "Es muy difícil pretender incluir a los pobres (...o a las personas con discapacidad) cuando no se sabe, o no se quiere saber, la manera por la cual fueron excluidos" (Demo, 1988).

En segundo lugar, la construcción social de la discapacidad (Medeiros \& Diniz, 2004) se nutre de la configuración de una identidad social caracterizada por la insuficiencia, la carencia y la falta de normalidad, de autonomía que es impuesta desde el entorno.

En tercer lugar, las prácticas en relación con la discapacidad se ubican en un lugar de especialización que despoja al sujeto de control y lo coloca en situación de dependencia.

Estos elementos dificultan la reflexión, la construcción y la acción en relación a la necesidad de garantizar socialmente oportunidades reales para capacidades y funcionamientos diferentes, a partir del reconocimiento de condiciones estructurales de desigualdad social, injusticia, explotación y la discriminación como razón de ser de un sistema de rehabilitación integral.

De otro lado, la problemática se complejiza si se tiene en cuenta que la posibilidad de acceder a los recursos socialmente disponibles está mediada por la capacidad que tienen los sectores subalternos de forzar procesos de redistribución en su favor (Hernández-Jaramillo \& Hernández-Umaña, 2005), así como por el poder efectivo de que disponen estos grupos para 
exigir la adopción de políticas públicas que propicien su integración social.

En esta línea, Fátima Correa (2013) llama la atención en algunas líneas de acción que buscan aportar al proceso de inclusión, tales como no reducir al otro a su condición de exclusión; decir no a la utopía del cuerpo perfecto y crear espacios de negociación para la persona, su familia, para la comunidad y para los servicios que se ocupan de ellos; ampliar la formación ética y política de profesionales de salud, educación, cultura, trabajo, arte, asistencia social, y ampliar la participación política de los profesionales y de la población con discapacidad.

\section{Formación profesional, reproducción de lógicas de dominación y exclusión}

Investigaciones previas han reseñado la necesidad de consolidar las competencias profesionales en rehabilitación para poder proveer intervenciones (no necesaria y exclusivamente clínicas) (Moreno-Angarita, et al., 2016). En términos de la práctica del talento humano en rehabilitación, se observa falta de delimitación normativa de las funciones según las profesiones involucradas en el proceso de rehabilitación y el rol poco claro de los profesionales en campos distintos al asistencial, con escasa cualificación para el ejercicio en ámbitos comunitarios, educativos, laborales y sociales. Parece que los profesionales no se asumieran como seres sociales, con la responsabilidad de contribuir a mejorar la calidad de vida de la sociedad en la cual viven. Se destacan la falta de una visión integral, interprofesional e intersectorial, acorde con las necesidades de los usuarios de los servicios de rehabilitación, así como el desconocimiento de las características y mecanismos de acceso al sistema para la atención a las personas.

En este marco, vale la pena destacar que la educación profesional, vista desde las teorías crítico - reproductivistas, cumple un papel de permanencia / aceptación del estado de cosas en relación a la división social del trabajo por grupos (propietarios / asalariados) y las relaciones de dominación / explotación / injustica que se derivan de ella. Así, la educación prepara al profesional para aceptar los arreglos sociales en términos de una organización y división del trabajo, unas lógicas de control y de fragmentación - descalificación que garantizan la productividad y la acumulación de capital en manos de actores sociales que no siempre son claramente reconocidos. Este proceso de aceptación / reproducción se finca en los siguientes dispositivos (Molina-Achury, 2015):

- Naturalización de las lógicas (no problematización) de dominación. En el proceso formativo se instalan y naturalizan la dominación por género $^{5}$, profesional, laboral, que imperan en la práctica profesional y en la relación docente - estudiante; lo que posteriormente contribuye a

\footnotetext{
${ }^{5}$ Como reconocimiento cultural, implica tener en cuenta la diversidad y el reconocimiento de identidades subvaloradas por el androcentrismo, la xenofobia, el heterosexismo y la violencia. Se viven segregaciones entre el estudiantado debido a la etnia, el desplazamiento forzado, la discapacidad, el fracaso escolar, las enfermedades.
} 
naturalizar las lógicas dominantes por clase social, condición de discapacidad, etnia, entre otras.

- El peso de la estructura de conocimiento médico y su reconocimiento social hegemoniza la construcción disciplinar, definiendo con frecuencia sus lenguajes y limitando prácticas concretas, y reproduciendo con ello la lógica biologista y de funcionamiento, así como los patrones de "normalidad - anormaidad".

- La presión de las lógicas instituidas de una objetividad declarada, fuente de verdad y ciencia que se retroalimenta cotidianamente con diversos mecanismos en la investigación, en las lógicas de publicación, en todo aquello que refiera legitimidad académica.

- La generación y apropiación del conocimiento aparece fragmentada. Se limita la posibilidad para el desarrollo disciplinar y profesional crítico como resultado de la presión que ejerce el interés del mercado, el cual define temas prioritarios, paradigmas válidos, métodos, e incluso para algunos, resultados en términos de nueva tecnología, que no necesariamente está vinculada con la efectividad y las necesidades.

- Distanciamiento de la realidad concreta, como fundamento del propio proceso educativo y que es reconocido como tardío en su aparición en el currículo. En el caso que nos ocupa, es clara la preocupación por favorecer un aprendizaje de técnicas y procesos en el marco de lógicas de ordenamiento y comportamiento instituidas, sin conflictividad, para lo cual la realidad se amolda a los interés del proceso educativo; con esto se debilita su posibilidad como elemento cuestionador.

- Las propias condiciones de trabajo docente y sus limitaciones se articulan con todos y cada uno de los aspectos mencionados. Es innegable que un análisis pormenorizado del mismo permite validar la idea de que en el ejercicio docente existe una permanente tensión por el desarrollo de un trabajo alienado, que se suma a la formación de trabajadores profesionalizados alienados: el docente no controla, no gobierna, no gestiona, no reconoce el proceso en su conjunto; al tiempo, instala unas habilidades en un sujeto que no domina la lógica del proceso, no está en capacidad de una interacción incidente o transformadora, y que opera mecánicamente unas habilidades. Se prepara para condicionarlos a una institucionalidad y a unas relaciones que ordenan el trabajo bajo la lógica de la productividad y que imponen paquetes restrictos de beneficios y de tiempos de atención, los cuales limitan la práctica profesional en lo que respecta al grado de autonomía, sujeto ahora por la racionalidad económica. Al tiempo, el profesional - trabajador, colocado en una lógica creciente de trabajador precarizado, enfrenta condiciones de trabajo cada vez más críticas, lo que restringe sus posibilidades de desarrollo como profesional, en el marco de un trabajo alienado que 
le limita en su desarrollo como ser humano.

En este contexto, la educación profesional tiene el reto de replantearse como posibilidad de transformación, siempre y cuando se problematice a sí misma, a partir de lo cual identifique trayectos y nuevas referencias de sentido.

\section{Elementos a considerar en la transformación del proceso formativo} (Molina-Achury, 2015)

La interacción profesional - sujeto tiene como reto afincar el proceso de formación en el reconocimiento de la diferencia como posibilidad de vida; a partir de allí, superar la fragmentación, individualización y descontextualización, buscando centralmente combatir todos los sentidos y prácticas relacionados a la discapacidad como fuente de inequidad.

Dentro de los aspectos que a partir de este análisis pueden ser colocados como referencial, se parte en primera instancia de la necesidad de establecer, como un sentido básico del proceso de formación, el reconocimiento y la crítica a toda forma de relación de dominación, sea esta por género, clase, etnia o discapacidad, en una perspectiva que los vincule. Las lógicas de poder, siguiendo a Mayo (2004), basado en Gramsci, plantean comprender su naturaleza no estática (está constantemente abierta a negociación y renegociación, por tanto, a ser renovada y recreada), incompleta, selectiva y con posibilidad de crisis, lo que indica que puede haber espacio para una acción contra hegemónica, en donde la educación y la formación cultural de adultos son claves.

Así mismo, se trata de cuestionar los contendidos de la cultura dominante, desde la apropiación crítica del conocimiento y la apropiación crítica y selectiva de la cultura dominante, y de abogar por una práctica que integre permanentemente reflexión y acción con capacidad de transformación, favoreciendo la articulación entre teoría y práctica, entre conciencia y acción, uno de los grandes vacíos en la formación profesional. Para esto, se rescata la necesidad de garantizar el vínculo con la realidad como fundamento educativo, con lo cual todos los escenarios de práctica deben ser asumidos como posibilidad para la democracia, a partir de una mayor comprensión del funcionamiento de la sociedad y del control sobre el proceso de trabajo, la identificación de los conflictos y de las posibilidades de resistencia y de cambio, de tal suerte que se oriente el proceso hacia la construcción de sujetos libres y con autodeterminación.

En este contexto, la coyuntura actual nacional del posacuerdo ha dado relieve al tema de discapacidad / rehabilitación, lo que se convierte en una oportunidad en un escenario de nuevas reformas y transformaciones del sistema de salud y las políticas sociales. Lo anterior, junto con las posibilidades y experiencias desarrolladas en torno a los modelos de la Atención Primaria en Salud, se planea como ventana de oportunidad para un proceso de replanteo de la formación profesional en torno a la rehabilitación. Estos escenarios hacen viable la posibilidad de 
materializar la idea de integralidad en una perspectiva cotidiana y cercana a las personas, incluso en términos de su territorialidad, con una visión que amplía la idea de rehabilitación a todos los ciclos y al conjunto de requerimientos en salud, retomando aprendizajes y enfoques de la Rehabilitación Basada en Comunidad y la Atención Primaria en Salud.

Siendo un aspecto central la construcción de relaciones democráticas y dialógicas, tanto en la formación como en la sociedad, es fundamental en el proceso educativo la vinculación con grupos más amplios, movimientos y partidos que representan la perspectiva de las personas con discapacidad y que cimenten la posibilidad de alianzas con fuerza incidente en las políticas vinculadas con la rehabilitación.

\section{Referencias}

Angarita, D.C. (2014). Rehabilitación integral: un análisis de contenido del marco normativo de apoyo a la discapacidad (Tesis de Maestría). Bogotá: Universidad Nacional de Colombia.

Buitrago, M.T. (2013). Discapacidades peregrinas. Construcciones sociales de la discapacidad en Colombia: aportes para la Salud Pública desde una perspectiva crítica (Tesis de doctorado). Bogotá: Universidad Nacional de Colombia.

Congreso de la República de Colombia. (2007). Ley 1145 de 2007. Por medio de la cual se organiza el Sistema Nacional de Discapacidad y se dictan otras disposiciones. Recuperado de: https://www. minsalud.gov.co/Normatividad_Nuevo/ LEY\%201145\%20DE\%202007.pdff

Corrêa-Oliver, F. (julio, 2013). Ponencia. En Seminario Rehabilitación Integral: aproxi- maciones teóricas y recomendaciones frente a su praxis. Universidad del Rosario, Bogotá, Colombia.

Departamento Nacional de Planeación. (2013). Documento Conpes Social 166. Política Pública Nacional de Discapacidad e Inclusión Social. Recuperado de: https://www.minsalud.gov.co/sites/rid/ Lists/BibliotecaDigital/RIDE/INEC/IGUB/ CONPES166.pdf

Demo, P. (1988). Participação é conquista. São Paulo: Cortez.

Gobierno de la República de Colombia. (2013). Ley 1618 de 2013. Por medio de la cual se establecen las disposiciones para garantizar el pleno ejercicio de los derechos de las personas con discapacidad. Recuperado de: http://wsp.presidencia.gov.co/Normativa/Leyes/Documents/2013/LEY\%201618\%20DEL\%20 $27 \% 20$ DE $\% 2$ OFEBRERO $\% 20$ DE $\% 20$ 2013.pdf

Hernández-Jaramillo, J. \& Hernández-Umaña, I. (2005). Una aproximación a los costos indirectos de la discapacidad en Colombia. Revista Salud Pública, 7 (2), 130-144.

Mayo, P. (2004). Gramsci, Freire e a educação de adultos: possibilidades para uma ação transformadora. Trad. Carlos Alberto Silveira Netto Soares. Porto Alegre: Artmed.

Medeiros, M. \& Diniz, D. (2004). A nova maneira de se entender a deficiência e o envelhecimento (Texto para Discussão № 1040). Instituto de Pesquisa Económica Aplicada, Brasil. Recuperado de: http:// bvsms.saude.gov.br/bvs/publicacoes/ td_1040.pdf

Ministerio de Salud Colombia. (2015). Discapacidad. Recuperado de: https://www. minsalud.gov.co/proteccionsocial/Paginas/DisCAPACIDAD.aspx

Molina-Achury, N. (2015). Educación en Fisioterapia: análisis crítico desde la práctica profesional (Tesis de doctorado). Sao Paulo: Universidad de São Paulo. 
Molina-Achury, N., Mogollón-Pérez, A., Balanta-Cobo, P., Moreno-Angarita, M., Hernández-Jaramillo, B., Rojas-Castillo, C.P. (2016). Lógicas dominantes en la formulación de políticas y organización de servicios que inciden en el acceso a la rehabilitación integral. Revista Gerencia y Políticas de Salud, 15 (30), 94-107.
Moreno-Angarita,M., Hernández-Jaramillo J., Balanta-Cobo, P., Mogollon-Pérez, A., Molina-Achury, N., Rojas Castillo, C. (2016, en prensa). Análisis cualitativo del concepto y praxis de rehabilitación integral percibido por distintos actores involucrados. En prensa Revista Facultad de Medicina, Universidad Nacional de Colombia. 